\title{
Review of "Addiction Paper Authoring Tool (PAT): A Guide"
}

Sharon Cox

1 London South Bank University

A thoroughly comprehensive overview of how the Paper Authoring Tool (PAT) can assist researchers. For studies with pre-defined outcomes and strict protocols (e.g., Randomised Control Trial, RCT), PAT will no doubt prompt the author to include the necessary and most useful information. As an example, for smoking cessation trials, one can be prompted for follow up at 4, 12 and 24 weeks, and this would lead to a prompt to define the outcome measure at each time point. Allowing and prompting for deviations from protocol is especially useful, and it is reassuring for researchers that deviations need not be a deal breaker for publication, if justified and fully explained.

However, I am wondering what the vision for PAT is in relation to qualitative articles? Firstly, in terms of the general construction of the main headings. Secondly, how the Addiction Ontology (and others, e.g., E-CigO) will underwrite PAT, where the structure and findings may be less fixed or predefined. Lastly, protocols may also be less fixed to allow for greater exploration within the context of the data collection, will this be considered? Some extra information regarding the utility of PAT for mixed method researchers may be required. 